\title{
Suitability of Indian potato genotypes for preparation of ready-to-serve canned curries
}

\author{
Sukhpreet Kaur and Poonam Aggarwal
}

Ready-to-eat canned potato curry made from three potato genotypes namely Kufri Chipsona-1, Kufri Chandramukhi and Kufri Pukhraj were evaluated for quality characteristics in order to determine their suitability for processing. Canned product was stored for a period of 6 months at room temperature to assess its shelf-life properties. Incubation test on the product was carried at $55^{\circ} \mathrm{C}$ for 45 days. Significant differences in physico-chemical parameters were observed among the genotypes. During storage of canned potato curries, all the quality parameters remained almost stable with the exception of ascorbic acid which decreased during storage, irrespective of genotypes. Physical conditions of cans were found to be satisfactory during storage. Storage study revealed that canned product can be safely stored for up to 6 months with high acceptability ratings. Also, product prepared from Kufri Pukhraj displayed excellent keeping quality during the entire storage period. Incorporation of such potato genotypes into low-cost value added products could serve as an excellent vehicle for enhancing the utilization of this resourceful food crop.

Key Words : Canning, Genotype, Potato, Processing, Shelf-life, Storage

How to cite this article : Kaur, Sukhpreet and Aggarwal, Poonam (2015). Suitability of Indian potato genotypes for preparation of ready-to-serve canned curries. Food Sci. Res. J., 6(1): 108-114.

Associate Authors' :

POONAM AGGARWAL, Department of Food Science and Technology, Punjab Agricultural University, LUDHIANA (PUNJAB) INDIA 